\title{
A METHOD FOR THE ASYMPTOTIC ESTIMATION OF INTEGRALS*
}

\author{
A. N. TIKHONOV, A. A. SAMARSKII and A. A. ARSEN'EV \\ Moscow
}

(Received 30 March 1972)

A METHOD for the asymptotic estimation of integrals with a kernel of $\delta$-function type is presented.

In $[1-3]$ in the solution of the problem of finding the asymptotic forms of definite integrals with a kernel of $\delta$-function type a special difficulty was encountered which consisted of the fact that the integration of the asymptotic expression for the integrand obtained leads to formally infinite coefficients of the asymptotic form of the integral. This situation is characteristic of many asymptotic problems.

A special method for the asymptotic estimation of integrals was developed in [1-3] to overcome this difficulty. The proposed method is based on the recurrence relations given in [1-3] and is applicable to many other problems, and for this reason it is useful to give an independent treatment of this method.

1. Asymptotic form of integrals over a finite interval

We first consider the problem of calculating the asymptotic form as $h \rightarrow 0$ of the integral

$$
I(h)=\frac{1}{h} \int_{0}^{1} \omega\left(\frac{x}{h}\right) f(x) d x .
$$

We will suppose that the kernel $\omega(x)$ and the function $f(x)$ satisfy the following conditions.

Condition 1. The function $\omega(x)$ is integrable in any finite interval and as $x \rightarrow \infty$ has the asymptotic form

* Zh, vychisl. Mat. mat. Fiz., 12, 4, 1005-1012, 1972. 


$$
\omega(x)=\sum_{n=1}^{m} \frac{q_{n}(x)}{x^{n}}+\bar{\omega}_{m}(x), \quad 1 \leqslant m \leqslant N
$$

where $x^{m} \bar{\omega}_{m}(x) \rightarrow 0$ as $x \rightarrow \infty$,

$$
\int_{a}^{\infty}\left|x^{m-1} \omega_{m}(x)\right| d x<\infty, \quad a>0 .
$$

We will assume that the functions $q_{n}(x)$ are integrable in any finite interval.

Condition 2. The function $f(x)$ has $N$ right derivatives at the point $x=0$, which are bounded and integrable in the interval $[0,1]$.

For every $m, 0 \leqslant m<N$, we define the functions

$$
\begin{aligned}
& F_{m+1}(x)=\frac{F_{m}(x)-F_{m}(0)}{x}, \quad F_{0}(x)=f(x), \\
& \omega_{m+1}(x)=x \omega_{m}(x)-q_{m+1}(x), \quad \omega_{0}(x)=\omega(x), \\
& I_{m}(h)=\frac{1}{h} \int_{0}^{1} \omega_{m}\left(\frac{x}{h}\right) F_{m}(x) d x \\
& C_{m}(h)=\int_{0}^{1} q_{m+1}\left(\frac{x}{h}\right) F_{m+1}(x) d x,
\end{aligned}
$$

(2)

$$
B_{m}(h)=\frac{1}{h} \int_{0}^{1} \omega_{m}\left(\frac{x}{h}\right) d x
$$

Conditions 1 and 2 ensure the correctness of the definitions (2), the following formulas holding:

(3)

$$
F_{m}(0)=\frac{f^{(m)}(0)}{m !}, \quad F_{m}(x)=\frac{1}{x^{m}}\left[f(x)-\sum_{n=0}^{m-1} \frac{f^{(k)}(0)}{k !} x^{k}\right],
$$

$$
\omega_{m}(x)=x^{m} \overline{\omega_{m}}(x)
$$

\section{Lemma 1}

The following estimate holds: 


$$
\left|h I_{m}(h)\right| \rightarrow 0 \text { as } h \rightarrow 0,0 \leqslant m<N .
$$

Proof. By Condition 1,

$$
x^{m} \bar{\omega}_{m}(x)=\omega_{m}(x) \rightarrow 0, x \rightarrow \infty
$$

therefore

and

$$
\frac{1}{x} \int_{0}^{x}\left|\omega_{m}(t)\right| d t \rightarrow 0, \quad x \rightarrow \infty
$$

$\left|h I_{m}(h)\right|=\left|\int_{0}^{1} \omega_{m}\left(\frac{x}{h}\right) F_{m}(x) d x\right| \leqslant \sup _{0<x<1}\left|F_{m}(x)\right| h \int_{10}^{1 / h}\left|\omega_{m}(t)\right| d t \rightarrow 0, \quad h \rightarrow 0$

\section{Theorem 1}

The following recurrence relations hold:

(5)

$$
I_{m}(h)=F_{m}(0) B_{m}(h)+C_{m}(h)+h I_{m+1}(h), \quad 0 \leqslant m<N, \quad h>0
$$

Indeed,

$$
\begin{aligned}
I_{m}(h)= & \frac{1}{h} \int_{0}^{1} \omega_{m}\left(\frac{x}{h}\right) F_{m}(x) d x=\frac{1}{h} \int_{0}^{1} \omega_{m}\left(\frac{x}{h}\right)\left[x F_{m+1}(x)+F_{m}(0)\right] d x= \\
& =F_{m}(0) B_{m}(h)+\frac{1}{h} \int_{0}^{1} \frac{\omega_{m+1}(x / h)+q_{m+1}(x / h)}{(x / h)} x F_{m+1}(x) d x= \\
& =F_{m}(0) B_{m}(h)+\int_{0}^{1} \omega_{m+1}\left(\frac{x}{h}\right) F_{m+1}(x) d x+ \\
& +\int_{0}^{1} q_{m+1}\left(\frac{x}{h}\right) F_{m+1}(x) d x=F_{m}(0) B_{m}(h)+C_{m}(h)+h I_{m+1}(h) .
\end{aligned}
$$

Therefore, equation (5) is proved. The relations (4) and (5) imply Theorem 2.

Theorem 2

If Conditions 1 and 2 are satisfied, the following as ymptotic estimate holds: 


$$
I(h)=\sum_{m=0}^{\pi-1}\left[F_{m}(0) B_{m}(h)+C_{m}(h)\right] h^{m}+h^{N-1} \rho(h), \quad \rho(h) \rightarrow 0, \quad h \rightarrow 0
$$

Formula (6) is fundamental in the proposed method of calculating the asymptotic form of the integrals. It enables the problem of the calculation of the asymptotic form of the integral (1) to be reduced to the calculation of the asymptotic forms of the integrals $B_{m}(h)$ and $C_{m}(h)$. We notice that the integral $B_{m}(h)$ depends only on the kernel $\omega(x)$, and the integral $C_{m}(h)$ only on the single coefficient $f(x)$ and the function $f(x)$.

As a first example we consider the case where all the coefficients $q_{m}(x)$ are constants:

$$
q_{m}(x) \equiv q_{m}=\text { const. }
$$

Then

$$
C_{m}(h)=q_{m+1} \int_{0}^{1} F_{m+1}(x) d x
$$

and the asymptotic value of the integrals $B_{m}(h)$ is calculated as follows:

Since

$$
\begin{aligned}
& B_{m}(h)=\int_{u}^{1 / h} \omega_{m}(t) d t=\int_{0}^{1} \omega_{m}(t) d t+\int_{i}^{1 / h}\left[\omega_{m}(t)-\frac{q_{m+1}}{t}\right] d t-q_{m+1} \ln h= \\
& =\int_{0}^{1} \omega_{m}(t) d t+\int_{i}^{\infty}\left(\omega_{m}(t)-\frac{q_{m+1}}{t}\right) d t-q_{m+1} \ln h+\int_{\infty}^{1 / h}\left(\frac{q_{m+2}}{t^{2}}+\ldots\right) d t .
\end{aligned}
$$

$$
\left|\int_{\infty}^{1 / h} t^{m} \bar{\omega}_{N}(t) d t\right| \leqslant h^{N-1-m} \int_{\infty}^{1 / h}\left|t^{N-1} \bar{\omega}_{N}(t)\right| d t=h^{N-1-m_{\rho}} \rho(h)
$$

we have

$$
\begin{aligned}
& B_{m}(h)=\int_{0}^{1} \omega_{m}(t) d t+\int_{1}^{\infty}\left(\omega_{m}(t)-\frac{q_{m+1}}{t}\right) d t- \\
& -q_{m+1} \ln h+q_{m+2} h+\frac{1}{2} q_{m+3} h^{2}+\ldots+h^{N-i-m} \rho(h), \quad h \rightarrow 0 .
\end{aligned}
$$

After substituting formula (7) in (5), we obtain the following theorem. 


\section{Theorem 3}

If the functions $\omega(x)$ and $f(x)$ satisfy Conditions 1 and 2 , where all the coefficients $q_{m}(x)$ are constants, the integral (1) has as $h \rightarrow 0$ the following asymptotic form:

$$
\begin{aligned}
& I(h)=\sum_{m=0}^{N-1}\left[-q_{m+1} \frac{f^{(m)}(0)}{m !} \ln h+\left(\int_{0}^{1} \omega_{m}(x) d x+\right.\right. \\
& \left.+\int_{1}^{\infty}\left(\omega_{m}(x)-\frac{q_{m+1}}{x}\right) d x\right) \frac{f^{(m)}(0)}{m !}+q_{m+1}\left(\int_{0}^{1} F_{m+1}(x) d x-\right. \\
& \left.\left.-\sum_{m=0}^{m-1} \frac{f(m)(0)}{k !(m-k)}\right)\right] h^{m}+h^{N-1} \rho(h), \quad \rho(h) \rightarrow 0, \quad h \rightarrow 0 .
\end{aligned}
$$

Formula (8) was first obtained in [1-2].

As a second example we consider the case where

$$
q_{m}(x)=\beta_{m}+\sum_{k=1}^{h_{m}} \beta_{k, m} x^{-\alpha_{k, m},} \quad 0<\alpha_{k, m}<1 .
$$

In this case

$$
C_{m}(h)=\beta_{m+1} \int_{0}^{1} F_{m+1}(x) d x+\sum_{k=1}^{k_{m+1}} \beta_{k, m+1} \hbar^{-\alpha_{k, m+1}} \int_{0}^{1} \frac{F_{m+1}(x)}{x^{\alpha_{k, m}}} d x
$$

and the asymptotic value of the integral $B_{m}(h)$ is easily calculated from the equation

$$
\begin{aligned}
& B_{m}(h)=\int_{0}^{1} \omega_{m}(x) d x+\int_{1}^{\infty}\left(\omega_{m}(x)-\frac{\beta_{m+1}}{x}\right) d x-\beta_{m+1} \ln h+ \\
& +\int_{\infty}^{1 / h}\left(\frac{q_{m+1}-\beta_{m+1}}{x}+\frac{q_{m+2}}{x^{2}}+\ldots\right) d x= \\
& =\int_{0}^{1} \omega_{m}(x) d x+\int_{1}^{\infty}\left(\omega_{m}(x)-\frac{\beta_{m+1}}{x}\right) d x-\beta_{m+1} \ln h+ \\
& +\sum_{k=1}^{h_{m}} \frac{\beta_{k, m+1}}{\alpha_{k, m+1}} h^{\alpha_{k, m+1}}+\beta_{m+2} h+\ldots
\end{aligned}
$$

The general formula for the last case is rather unwieldy to obtain and we 
will not write it out.

We specially mention the case where $q_{1}=$ const and the function $f(x)$ is continuous only on the right. Then reasoning similar to that given above enables us to state that

$$
I(h)=\left[-q_{1} \ln h+\int_{0}^{1} \omega(x) d x+\int_{1}^{\infty}\left(\omega(x)-\frac{q_{1}}{x}\right) d x\right] f(+0)+o(1)
$$

\section{Asymptotic form of integrals over an infinite interval}

1. General case. We now consider integrals of the form

$$
I(h)=\frac{1}{h} \int_{0}^{\infty} \omega\left(\frac{x}{h}\right) f(x) d x .
$$

We suppose that the function $\omega(x)$ satisfies the following condition.

Condition 3. The function $f(x)$ is integrable over any finite interval, is bounded on $[0, \infty)$, and the integrals

$$
\left|\int_{1}^{\infty} q_{m+1}\left(\frac{x}{h}\right) \frac{f(x)}{x^{m}} d x\right|<\infty, \quad 0 \leqslant m<N .
$$

are finite for any $h>0$.

The following theorem holds.

\section{Theorem 4}

If Conditions $1-3$ are satisfied, the asymptotic estimate

$$
\begin{aligned}
& \left.I(h)=\sum_{\substack{m=0 \\
+h^{N-1} \rho(h) .}}^{N-1} \boldsymbol{F}_{m}(0) B_{m}(h)+C_{m}(h)+\int_{1}^{\infty} q_{m+1}\left(\frac{x}{h}\right) f(x) x^{-m} d x\right] h^{m}+ \\
&
\end{aligned}
$$

holds for the integral (9).

Proof. We divide the integral (9) into the two integrals:

$$
I(h)=\frac{1}{h} \int_{0}^{1} \omega\left(\frac{x}{h}\right) f(x) d x+\frac{1}{h} \int_{1}^{\infty} \omega\left(\frac{x}{h}\right) f(x) d x .
$$


In the second integral we replace the kernel $\omega(x)$ by its asymptotic estimate, and we find the asymptotic estimate of the first integral by formula (6). If all the coefficients $q_{m}(x)$ are constants (the case considered in $[1,2]$ ), we obtain from (8) and (10) that the following theorem holds.

Theorem 5

If the conditions of Theorem 3 are satisfied, and $f(x)$ is integrable and bounded on $[1, \infty)$, the following estimate holds for the integral (9):

$$
\begin{aligned}
& I(h)=\sum_{m=0}^{N-1}\left[-\left(q_{m+1} \ln h+\int_{0}^{1} \omega_{m}(x) d x+\int_{1}^{\infty}\left(\omega_{m}(x)-\frac{q_{m+1}}{x}\right) d x\right) \times\right. \\
& \left.\times \frac{f^{(m)}(0)}{m !}+q_{m+1} P_{m}(f)\right] h^{m}+h^{N-1} \rho(h), \quad \rho(h) \rightarrow 0, \quad h \rightarrow 0, \\
& P_{m}(f)=\int_{0}^{1}\left[f(x)-\sum_{k=0}^{m-1} \frac{f^{(k)}(0)}{k !} x^{k}\right] x^{-m} d x+\int_{1}^{\infty} \frac{f(x)}{x^{m}} d x-\sum_{k=0}^{m-1} \frac{f^{(h)}(0)}{k !(m-k)} .
\end{aligned}
$$

If the function $f(x)$ has $N$ continuous derivatives in the interval $[0, \infty)$, and

$$
\left|x^{N} f^{(m)}(x)\right| \rightarrow 0, \quad x \rightarrow \infty, \quad 0 \leqslant m<N
$$

the functional $P_{m}(f)$ assumes the form

$$
P_{m}(f)=\frac{1}{m !}\left[\int_{0}^{1} \frac{f^{(m)}(x)-f^{(m)}(0)}{x} d x+\int_{1}^{\infty} \frac{f^{(m)}(x)}{x} d x+f^{(m)}(0) \sum_{\nu=1}^{m} \frac{1}{v}\right] .
$$

2. A special case. If for every $m$ the integrals

$$
B_{m}^{*}=\int_{0}^{\infty} \omega_{m}(x) d x
$$

are finite, we also use another method for calculating the asymptotic value of the integral (9). We put

$$
I_{m}^{*}(h)=\frac{1}{h} \int_{0}^{\infty} \omega_{m}\left(\frac{x}{h}\right) F_{m}(x) d x, \quad C_{m}^{*}(h)=\int_{0}^{\infty} q_{m+1}\left(\frac{x}{h}\right) F_{m+1}(x) d x
$$

It is easy to see that the integrals introduced exist and are connected by the recurrence formula 


$$
I_{m}^{*}(h)=\frac{f^{(m)}(0)}{m !} B_{m}^{*}+C_{m}^{*}(h)+h I_{m+1}^{*}(h)
$$

To prove formula (12) it is sufficient to prove the convergence for every $m$ of the integral $I_{m}{ }^{*}(h)$, since then the recurrence formula itself and the convergence of the integral $C_{m}{ }^{*}(h)$ will be consequences of the recurrence relations between the functions $\omega_{m}(x), q_{m}(x), F_{m}(x)$. However, because of the boundedness of the function $f(x)$ for every $h>0$ the estimate

$$
F_{m}(h x)=O(1 / x h), x \rightarrow \infty \text {, }
$$

is valid, and the integral $\int_{a}^{\infty} \frac{\left|\omega_{m}(x)\right|}{x} d x, \quad a>0$, converges by Condition 1 . We
now prove that

$$
|h|_{N} *(h) \mid \rightarrow 0 \text { as } h \rightarrow 0 \text {. }
$$

Indeed, by (13) we have

$$
\begin{aligned}
& \left|h I_{N}{ }^{*}(h)\right|=\left|h \int_{0}^{a} F_{N}(h t) \omega_{N}(t) d t+\int_{u}^{\infty}\left(h t F_{N}(h t)\right) \frac{\omega_{N}(t)}{t} d t\right| \leqslant \\
& \leqslant h \int_{i}^{a}\left|F_{N}(h t)\right|\left|\omega_{N}(t)\right| d t+\max _{a \leqslant t<\infty} h t\left|F_{N}(h t)\right| \int_{a}^{\infty} \frac{\left|\omega_{N}(t)\right|}{t} d t \leqslant \\
& \leqslant c_{1} h_{0}^{a}\left|\omega_{N}(t)\right| d t+c_{2} \int_{a}^{\infty} \frac{\left|\omega_{N}(t)\right|}{t} d t, \quad c_{1}=\text { const } \geqslant 0, \quad c_{z}=\text { const }>0 .
\end{aligned}
$$

First choosing $a$ sufficiently great, and then $h$ sufficiently small, we can make the right side of $\left(14^{\prime}\right)$ less than any positive $\epsilon$. Theorem 6 follows from (12), (14).

\section{Theorem 6}

If Condition 1-3 are satisfied and the integrals

$$
\int_{0}^{\infty} \omega_{m}(x) d x, \quad m=0,1, \ldots
$$

are finite, the following estimate is valid:

$$
I(h)=\sum_{m=0}^{N-1}\left[\frac{f^{(m)}(0)}{m !} B_{m}^{*}+C_{m}^{*}(h)\right] h^{m}+h^{N-1} \rho(h), \quad \rho(h) \rightarrow 0 \quad \text { as } \quad h \rightarrow 0 .
$$


As an example we consider the integral $I(h)$ whose kernel has as $x \rightarrow \infty$ the asymptotic form

$$
\omega(x)=\frac{e^{i x}}{\gamma x}\left(a_{1}+\frac{a_{2}}{x}+\ldots\right)
$$

Here

$$
q_{m}(x)=a_{m} \gamma x e^{i x}, \quad \omega_{m}(x)=a_{m+1} \frac{e^{i x}}{\gamma x}+O\left(\frac{1}{\gamma / x^{3}}\right), \quad x \rightarrow \infty,
$$

and formula (15) is applicable. In our case the coefficients $C_{m}(h)$ are given by the integrals

$$
C_{m}(h)=a_{m+1} h^{-1 / 2} \int_{0}^{\infty} e^{i x / h} \sqrt{ } x F_{m+1}(x) d x
$$

the asymptotic forms of which are easily obtained from known formulas (see [3], p. 61) or directly from the recurrence relations

$$
\begin{aligned}
& \int_{0}^{\infty} \frac{e^{i x / h}}{\gamma x} f(x) d x=\int_{0}^{\infty} \frac{e^{i x / h}}{\gamma / x}[f(x)-f(0)] d x+f(0) \gamma(\pi i h)= \\
& =f(0) \gamma(\pi i h)+i h \int_{0}^{\infty} \frac{e^{i x / h}}{\gamma x}\left[f^{\prime}(x)-\frac{1}{2} \frac{f(x)-f(0)}{x}\right] d x .
\end{aligned}
$$

Simple calculations give

$$
C_{m}(h)=a_{m+1} \gamma(\pi i)\left[f^{(m)}(0)+\sum_{k=1}^{N-m-1} \frac{0.5 \cdot 1.5 \ldots(k-0.5)}{k !} f^{(h+m)}(0)(i h)^{h}\right]+h^{N-m-0.5} \rho(h) .
$$

Substituting this formula in (15), we obtain (see [4]) the following theorem.

\section{Theorem 7}

If the function $f(x)$ satisfies the Conditions $1-3$ and the kernel $\omega(x)$ has the asymptotic form (16), the integral (9) has the following asymptotic form as $h \rightarrow 0$ :

$$
\begin{aligned}
I(h) & =\sum_{m=0}^{N-1} D_{m} \frac{f^{(m)}(0)}{m !} h^{m}+h^{N-1} \rho(h), \quad \rho(h) \rightarrow 0, \quad h \rightarrow 0, \\
D_{m} & =\int_{0}^{\infty} \omega_{m}(x) d x+\gamma(\pi i) \sum_{h+j=m} 0.5 \cdot 1.5 \ldots(k-0.5) i^{h} a_{j+1}+\gamma(\pi i) a_{m+1} .
\end{aligned}
$$


Applying this formula to the kernel $\omega(x)=J_{v}(x)$, where $J_{v}(x)$ is a Bessel function of order $\nu$, we obtain the asymptotic form of the Hankel transformation of the function $f(x)$ for large values of the argument $z=1 / h$ :

$$
\int_{0}^{\infty} J_{v}\left(\frac{x}{h}\right) f(x) d x=\sum_{m=0}^{N-1} Q_{m}(v) f^{(m)}(0) h^{m}+h^{N-1} \rho(h)
$$

where the polynomials $Q_{m}(\nu)$ are calculated by the recurrence formula

$$
\begin{aligned}
& Q_{0}(v)=1, \quad Q_{m}(v)=\frac{1}{m+1} \sum_{n=0}^{m} \frac{(-1)^{m+n+1}}{(m-n) !} Q_{n}(v) b_{n-m}^{(v)}, \\
& b_{n}^{(v)}= \begin{cases}(-1)^{p+1} n ! & \text { for } n=2 p+1, \\
n ! \frac{1 \cdot 3 \ldots(n-1)}{2^{p} p !} v & \text { for } n=2 p .\end{cases}
\end{aligned}
$$

We give some of the first of the polynomials $Q_{m}(v): Q_{0}(v)=1, Q_{1}(v)=v, Q_{2}(v)$ $=1 / 2\left(v^{2}-1\right), Q_{3}(v)=1 / 6\left(v^{3}-4 v\right), Q_{4}(v)=1 / 24\left(v^{4}-10 v^{2}+9\right)$. We mention that for $\nu=0$ formula (17) was obtained in [5].

The methods of $[1-3]$ were also used in $[6,7]$.

\section{Some generalizations}

1. We consider integrals of the form

$$
I(h)=\frac{1}{h} \int_{a}^{b} \omega\left(\frac{x-x_{0}}{h}\right) f(x) d x, \quad a<x_{0}<b .
$$

By a change of variables this integral is reduced to the sum of the two integrals:

$$
\begin{aligned}
& I(h)=\frac{x_{0}-a}{h} \int_{0}^{1} \omega\left(-\frac{\left(x_{0}-a\right)}{h} t\right) f\left(x_{0}+\left(a-x_{0}\right) t\right) d t+ \\
& +\frac{b-x_{0}}{h} \int_{0}^{1} \omega\left(\frac{b-x_{0}}{h} t\right) f\left(x_{0}+\left(b-x_{0}\right) t\right) d t .
\end{aligned}
$$

If the point $x_{0}$ is fixed, the asymptotic value of each of these two integrals can be calculated by the formulas given above. But if the point $x_{0}$ is close to $a$ or $b$, that is, $x_{0}-a \sim h$ or $b-x_{0} \sim h$, the parameter $\left(\left(x_{0}-a\right) / h\right)^{-1}$ or $\left(\left(b-x_{0} 1 / 2 /\right.\right.$ $h)^{-1}$ will no longer be small, but then an expansion of the function $f(t)$ in the 
neighbourhood of $x_{0}$ can be used. Corresponding formulas uniform with respect to $x_{0}$ were obtained in [8].

2. We consider an integral over the multidimensional domain $\Omega$ :

$$
I(h)=\frac{1}{h} \int_{\Omega} \omega\left(\frac{x-x_{0}}{h}\right) f(x) d x .
$$

First locating the coordinate origin at the point $x_{0}$ and changing to polar coordinates, we obtain

$$
\begin{aligned}
& I(h)=\int d \varphi_{1} \ldots d \varphi_{n-1}\left[\int_{0} \omega\left(\varphi_{1}, \ldots, \varphi_{n-1} ; \frac{r}{h}\right) \times\right. \\
& \left.\times f\left(\varphi_{1}, \ldots, \varphi_{n-1} ; r\right) r^{n-1} d r\right]
\end{aligned}
$$

the asymptotic form of the integral (18) can be obtained by integrating with respect to $\left(\phi_{1}, \ldots, \phi_{n-1}\right)$ the expansion of the inner integral (if the point $x_{0}$ is situated on the boundary the formulas of [9] must be used).

Translated by J. Berry

\section{REFERENCES}

1. TIKHONOV, A. N. and SAMARSKII, A. A. The expansion with respect to a parameter of integrals with a kernel of $\delta$-function type. Nauchn. dokl. Vyssh. shkoly. Ser. fiz.-matem. Nauk, 1, 54-61, 1959.

2. TIKHONOV, A. N. and SAMARSKI, A. A. The asymptotic expansion of integrals with a slowly decreasing kernel. Nauchn. dokl. Vyssh. shkoly. Ser. fiz.-matem. Nauk, 1, 62-69, 1959.

3. TIKHONOV, A. N. and SAMARSKII, A. A. Asymptotic expansion of integrals with a slowly decreasing kernel. Dokl. Akad. Nauk SSSR, 126, 1, 26-29, 1959.

4. ERDELYI, A. Asymptotic expansions (Asimptoticheskie razlozheniya) Fizmatgiz, Moscow, 1962.

5. TIKHONOV, A. N. Asymptotic behaviour of integrals containing Bessel functions. Dokl. Akad. Nauk SSSR, 125, 5, 982-985, 1959.

6. RIEKSTYN'SH, E. YA. Asymptotic representation of some integrals of convolution type. Latviiskii matem. ezhe godnik, 8, 223-239, 1970.

7. RIEKSTYN'SH, E. YA. Asymptotic expansions of integrals with kemels close to power or exponential functions. Latviishii matem, ezhegodnik, 10, 1971.

8. RAKHMATULINA, A. KH. Expansion, uniform in a segment, of an integral with decreasing kemel. Zh. vychisl. Mat. mat. Fiz., 9, 1, 185-194, 1969.

9. ARSEN'EV, A. A. The Fourier transformation of a slowly decreas ing function. Dokl. Akad. Nauk SSSR, 154, 2, 251-253, 1964. 